\title{
2 Korpus und Methode
}

\author{
Materialsammlung, Analyseverfahren und \\ Untersuchungsdesign
}

\subsection{Untersuchungsmaterial}

Betrachtet man die grafischen Kommunikationsmittel, die heutzutage im Umlauf sind, so findet sich eine beinahe übergangslose Mischung von hochprofessioneller Gestaltung hin zu reiner Laiengestaltung. In kaum einem Feld oder Einsatzbereich der visuellen Gestaltung finden sich heute ausschließlich professionell gestaltete Erzeugnisse. Seien es nun Corporate Design, Signaletik, Werbegrafik, Editorial Design oder Webgestaltung: Visitenkarten, Ladenbeschriftungen, Hinweisschilder, Werbebroschüren, Eventplakate und Websites werden immer wieder auch von gestalterischen Laien entworfen. Aufgrund der in Teil 1 beschriebenen 〈Demokratisierung〉 der grafischen Hilfsmittel (5.5.5) entstehen auch Hybridund Zwischenformen: Autodidakten mit etwas mehr Know-how gestalten Flyer oder Plakate mithilfe von professionellen Gestaltungsprogrammen; typografisch geschulte, jedoch konzeptuell weniger gut ausgebildete Polygrafinnen entwerfen in Druckereien Inserate oder Prospekte; Laien gestalten ihre Visitenkarten und Websites mittels frei oder billig verfügbarer professioneller 〈Templates`. Das für diese Arbeit gewählte Untersuchungsmaterial sollte dieses breite Spektrum der Elaborationsgrade grafischer Gestaltung spiegeln und insbesondere auch die Vielfalt der existierenden Amateurgestaltung und wenig elaborierten Gestaltung in den Blick bringen. Da es unmöglich gewesen wäre, alle Bereiche des Grafikdesigns einer fundierten Analyse zu unterziehen, musste die Auswahl thematisch und formal, aber auch räumlich und zeitlich eingegrenzt werden.

Die Materialrecherche sollte sich erstens auf visuelle Kommunikationsmittel aus den Bereichen der Gemeinwesenarbeit und der Lokalpolitik beziehen, da hier ein größerer Anteil an Laienarbeiten und wenig elaborierten grafischen Produkten zu erwarten war. Unter «Gemeinwesenarbeit` wird dabei die nicht-kommerzielle, auf die Stabilisierung oder Aktivierung der Gemeinschaft und des Zusammenlebens ausgerichtete Arbeit behördlicher, kirchlicher oder als Vereine oder Verbände organisierter Fachstellen, Zentren und Kommissionen verstanden. Neben der klassischen Stadteil- bzw. Quartierarbeit existiert auch Gemeinwesenarbeit im ländlichen Raum (vgl. Rohrmoser 2013). Als «lokalpolitische〉 Akteure werden vornehmlich die vor Ort agierenden politischen Parteien sowie weitere auf Stadt- oder Ortsräume fokussierte, politisch aktive Gruppierungen und Vereinigungen betrachtet. Gemeinwesenarbeit in der Tradition des «Community Organizing> überschneidet sich teilweise mit der lokalen Politik, da die Aktivierung 
der Bevölkerung eines Sozialraums aus dieser Sicht nicht staatlich, sondern via Bürgerinitiativen, Gewerkschaften oder Vereine geschieht (vgl. Alinsky 1946; Mohrlok et al. 1993). Im deutschsprachigen Raum versteht man Gemeinwesenarbeit dagegen meist als Bestandteil der 〈Sozialen Arbeit〉 (vgl. Galuske 2011; Hinte et al. 2007), die behördlich initiiert und organisiert und somit nicht unbedingt politisch motiviert ist. Überschneidungspunkte kann es auch hier geben, wenn Sozialarbeiterinnen und -arbeiter mit lokalen Parteien und Initiativen zusammenarbeiten oder politische und behördliche Akteure in denselben Kommissionen beschäftigt sind.

Um das Korpus aus den beiden Bereichen Gemeinwesenarbeit und Lokalpolitik weiter einzugrenzen, wurden zweitens ausschließlich gedruckte, analog vorliegende Kommunikationsmittel ausgewählt. Digitale und zeitbasierte Medien wie Websites oder Videos, die ebenfalls Anteile grafischer Gestaltung aufweisen, wurden ausgeschlossen, da sich ihre Gestaltungs- und Elaborationskriterien noch einmal stark von jenen analoger Medien unterscheiden. Drittens wurde die Materialsuche auf drei politische Gemeinden im Kanton Bern (Schweiz) eingeschränkt. Die ausgewählten Gemeinden sollten unterschiedlich groß sein, sowohl den städtischen, vorstädtischen als auch ländlichen Bereich abdecken und während des Untersuchungszeitraums Lokalwahlen durchführen: Bern (Stadt), Zollikofen (Agglomeration) und Schüpfen (Land). Die Recherche wurde viertens auf die Zeitspanne eines Jahres, 2012, limitiert.

\subsubsection{Visuelle Kommunikation in der Gemeinwesenarbeit und Lokalpolitik}

Die visuelle Kommunikation auf Ebene der Gemeinwesenarbeit oder der Lokalpolitik wurde bis heute kaum erforscht. Das Problem der Gestaltung von Informationen im Gemeinwesen, d.h. die Frage, mit welchen visuellen Mitteln man die Menschen eines Sozialraums überhaupt erreicht, emotional, rational und werteorientiert anspricht oder zur Partizipation anregt, wird weder in der soziologischen noch sozialpädagogischen Literatur aufgegriffen. Zwar werden Methoden und Handlungskonzepte der Gemeinwesenarbeit und Möglichkeiten der Aktivierung der Anwohner vorgestellt (z.B. Spitzenberger 2011), die dabei verwendeten visuellen Kommunikationsmittel oder Formen der Kontaktaufnahme und Information werden jedoch nicht thematisiert. Generell wird die Wichtigkeit ästhetischer Praxis in der Sozialarbeit erwähnt (Jäger/Kuckhermann 2004), jedoch nicht hinsichtlich visueller Kommunikationsmittel. Lediglich der Einsatz von Musik, bildender Kunst und Neuen Medien in der Arbeitspraxis wird in den Blick genommen, womit eher die Rolle der ästhetischen Erziehung sowie Kunst- und Gestaltungspädagogik angesprochen ist (vgl. Hoffmann et al. 2004) als jene der visu- 
ellen Kommunikation. Untersuchungen mit Fokus auf visuelle Gestaltung oder gar die Rolle, welche Laiengestaltung innerhalb der Gemeinwesenarbeit innehat, scheinen noch keine zu existieren.

Auch spezifisch auf die lokalpolitische visuelle Kommunikation ausgerichtete Studien finden sich nur spärlich. «Politische Kommunikation` allgemein ist zwar ein wichtiger Bereich sowohl der Politik- und Sozialwissenschaften als auch der Medien- und Publizistikwissenschaft (z.B. Jarren/Donges 2011; Rhomberg 2009; Schemer/Wirth/Wünsch 2010) und auch die Kommunikation der Lokal- oder Kommunalpolitik wird explizit untersucht (z.B. Ladner 2008, 2011; Wollmann/ Roth 1999). Im Bereich der visuellen politischen Kommunikation sind jedoch fast nur politische Plakate Gegenstand der Forschung (z.B. Geise 2011; Müller 1997; Podschuweit 2007). Neben der Wahlkampfkommunikation ist in der Schweiz auch die visuelle Abstimmungskommunikation von Bedeutung und erhält entsprechende Aufmerksamkeit in wissenschaftlichen Studien (z.B. Demarmels 2009). Aus Sicht der Visuellen Rhetorik wurden sowohl Schweizer Abstimmungsplakate (z.B. Arnold 2007) als auch das Plakat im Allgemeinen untersucht (z.B. Deiters 2005). Auch wurde bereits früh auf die rhetorische Wirksamkeit der visuellen Propaganda in verschiedenen politischen Systemen aufmerksam gemacht (Margolin 1979). Insgesamt lässt sich in der vorhandenen Forschungsliteratur zur visuellen politischen Kommunikation ein Fokus auf vorzeigenswerte, zeitlose Beispiele «großer Plakatkünstler〉 (in der Schweiz z.B. Niklaus Stöcklin, Theo Ballmer oder Stephan Bundi) feststellen und es werden fast ausschließlich Aspekte des Bildinhalts oder visuell-rhetorischer Figuren untersucht. Die Rolle unterschiedlicher Ausarbeitungsformen und -typen politischer Kommunikationsmittel wird nicht berücksichtigt. Ebenso wenig wird politische Alltags- und Laiengestaltung besprochen. Eine Ausnahme bildet hier eine Untersuchung politischer Graffitis (Klee 2010) - ein amateurgestalterisches Phänomen, das jedoch außerhalb des Untersuchungsfelds dieser Arbeit liegt. Für die Untersuchung der Wirkungsweisen, die aus dem gestalterischen Elaborationsgrad visueller Kommunikation in der Gemeinwesenarbeit und Lokalpolitik resultieren, kann also kaum auf Forschungsergebnisse zurückgegriffen werden, weshalb hier ein direkter Blick auf den Untersuchungsgegenstand methodisch sinnvoll erschien.

\subsubsection{Materialsammlung und Bestimmung des Korpus}

In Zusammenarbeit mit verschiedenen Akteuren der Gemeinwesenarbeit und Lokalpolitik - insbesondere Gemeindeverwaltungen, Quartierarbeit, politischen Parteien, Vereinen und Kirchgemeinden - aus den ausgewählten drei Gemeinden Bern, Zollikofen und Schüpfen wurde versucht, das Kommunikationsmaterial 
des Jahres 2012 möglichst umfassend zusammenzutragen, welches zur Bewerbung und Bekanntmachung von Anlässen und Angeboten der dort ansässigen Institutionen des Gemeinwesens verbreitet wurde bzw. für die im Herbst in allen drei Gemeinden stattfindenden Lokalwahlen und weitere politische Aktivitäten in Umlauf gebracht wurde. Zu den gesammelten Artefakten gehörten sämtliche visuellen Kommunikationsmedien, die gedruckt, ausgedruckt oder fotokopiert vorlagen: Flugblätter, Prospekte, Plakate, Broschüren, Faltblätter, Zeitungen, Postkarten, Inserate, Sonderformate (z.B. Visitenkarten, Buchzeichen, Lose) und bedruckte «Give-Aways». Die großformatigen Wahlplakate wurden schließlich aus dem Untersuchungskorpus ausgeschlossen, da ihre Rhetorik bereits relativ gut erforscht ist und sich zeigte, dass die Plakate in ihrer Elaborationsweise stark mit den offiziellen Wahlprospekten korrespondierten, aber auch weil sie für die gewählte Analysemethode der Auslegeordnung (siehe 2.3.2) zu unhandlich gewesen wären. Ebenso wenig wurden die lokalpolitischen Kleininserate berücksichtigt, die in Lokalzeitungen und Quartieranzeigern abgedruckt waren, da diese elaborativ im Kontext des jeweiligen Printprodukts $\mathrm{zu}$ bewerten wären. Auch die dreidimensionalen grafischen Sonderformate (z.B. Würfel) und Spezialanfertigungen (z.B. Taschentücher, Kekse, Kugelschreiber) wurden nicht ins Untersuchungskorpus aufgenommen. Denn sie stellen Gestaltungsformate für sich dar, die gesondert zu analysieren wären.

Grundsätzlich wurde innerhalb des so gesteckten thematischen, medialen, räumlichen und zeitlichen Rahmens Vollständigkeit und Repräsentativität angestrebt, doch zum einen waren einige Institutionen der betreffenden Gemeinden nicht zur Kooperation bereit, andere hatten nicht mehr alle produzierten Materialien des Jahrs 2012 greifbar, so dass gewisse Lücken im Material unvermeidbar waren. In der Stadt Bern musste die Sammlung im Bereich Gemeinwesenarbeit aufgrund der dezentralen Organisation und der schier unzählbaren Akteure auf den Stadtteil 3 konzentriert werden. Zum anderen stellten besonders engagierte Akteure ausführliches, teils redundantes Kommunikationsmaterial zur Verfügung, weshalb zur Wahrung der Verhältnismäßigkeit einige der gesammelten Beispiele weggelassen werden mussten. Am Ende der Materialsammlung und -eingrenzung umfasste das Korpus insgesamt rund 400 Artefakte unterschiedlicher Elaborationsgrade (siehe die Übersicht des Gesamtkorpus im Anhang). 


\subsection{Analysemethode}

\subsubsection{Rhetorische Designanalyse: Die wirkungsintentionale Analyse von Gestaltung}

Die rund 400 grafischen Artefakte aus Gemeinwesenarbeit und Lokalpolitik der Gemeinden Bern, Zollikofen und Schüpfen wurden ausgewiesenen Gestaltungsexpertinnen und -experten vorgelegt, um sie mit der Methode der «Rhetorischen Designanalyse` zu untersuchen. Die Rhetorische Designanalyse ist eine Methode der wirkungsintentionalen Analyse von Gestaltung und beruht auf der Annahme, dass in allen gestalteten Artefakten, also im professionellen Grafikdesign ebenso wie in der Amateurgrafik, bestimmte Wirkungsabsichten der Produzentinnen und Produzenten plausibel anzunehmen sind, die zur tatsächlich realisierten Wirkung in Beziehung gesetzt werden können. Die Rhetorische Designanalyse bewertet nicht in erster Linie, ob das Design 〈gut〉 oder «schlecht ist, sondern vielmehr, ob es in sich konsistent ist, ob es die intendierten Wirkziele erreicht, ob es rhetorisch angemessen kommuniziert, ob es erfolgreich ist. Diese Parameter sind andere als in herkömmlichen Modellen der Designanalyse, wie etwa der Produktsemantik, der Marktforschung oder wahrnehmungspsychologischen Ansätzen. Die Grundlage der Rhetorischen Designanalyse bilden die wahrnehmbaren Faktoren des Artefakts bzw. der intersubjektiv feststellbare visuelle (bzw. haptische, olfaktorische, akustische) Eindruck, den es bei Betrachtern oder Anwenderinnen hinterlässt. Dieses Analyseverfahren wurde im Verlauf mehrerer Forschungsprojekte am Forschungsschwerpunkt Kommunikationsdesign (heute: Institute of Design Research) der Hochschule der Künste Bern erarbeitet und weiterentwickelt, in denen die Autorin als wissenschaftliche Mitarbeiterin bzw. Projektleiterin beteiligt war (vgl. Teil 1, 4.4).

Erweitert auf die in dieser Arbeit interessierenden Fragestellungen der Elaboration und Imperfektion ergeben sich folgende leitende Fragen für die Rhetorische Designanalyse: Welche formalästhetischen Merkmale und Stilmittel weist das Artefakt auf? Welche Elaborationsmerkmale werden vorgefunden? Wie wirkt das Artefakt auf rationaler, emotionaler und ethischer Ebene? Auf welcher Stilhöhe agiert die Gestaltung? Wirkt das Artefakt professionell oder laienhaft? Ist es gekonnt oder dilettantisch ausgearbeitet? Welche Zusammenhänge lassen sich zwischen formaler Ausarbeitung und wahrgenommener Wirkung erkennen? Auf welche Wirkungsintention kann geschlossen werden? Sind Form und Elaborationsweise angemessen angesichts der angestrebten Wirkung? Die Rhetorische Designanalyse lässt sich schematisch in sechs Schritte aufteilen, die bei der Durchführung nicht immer strikt voneinander getrennt werden können: 
1. Formale Analyse: Darstellung der verwendeten Stilmittel und Kontextinformationen, produktionstechnische Imperfektion

2. Wirkungsanalyse: Beschreibung der wahrgenommenen Wirkungen, Wirkungsdimensionen (Logos-Ethos-Pathos), Stilhöhe (niedrig-mittel-hoch), wahrgenommener Elaborationsgrad (professionell, laienhaft, gekonnt etc.)

3. Wirkregeln: Korrelation von verwendeten Stilmitteln, Elaborationsweisen und Wirkung

4. Wirkungsintention: Angenommene Wirkziele und Verwendungszweck

5. Angemessenheit: Konfrontation mit dem Konzept des Aptum, Herausarbeiten kontraintentionaler Faktoren und Definition des möglichen Gestaltungsspielraums

6. Extrapolation: Anwendung auf Neugestaltungen

Der sechste Schritt bildet bereits die Überleitung von der Analyse in die Designpraxis - wobei noch einmal die enge Verschränkung von Theorie und Praxis in der Designrhetorik sichtbar wird. Der Übergang von den Wirkungshypothesen zur praktischen Umsetzung wird im Rahmen der designbasierten Studie genauer untersucht (s. Kapitel 6 in diesem Teil). Für eine qualifizierte Durchführung der Rhetorischen Designanalyse wurde das Erfahrungswissen professioneller Gestalterinnen und Gestalter mit Lehr- und Forschungserfahrung im Bereich des Kommunikationsdesigns in Anspruch genommen. Wie sprechen ausgewiesene Experten der visuellen Kommunikation über die gesammelten grafischen Artefakte? Die Methode der Rhetorischen Designanalyse wurde hierfür an in der gestalterischen Praxis erprobte Arbeitsschritte wie das «Rebriefing〉 und die «Diskussion in Anbetracht des Artefakts> angepasst. Da das Untersuchungsmaterial zu umfangreich war, um alle Artefakte im Detail analysieren zu können, wurden zwei aufeinanderfolgende Verfahren definiert, was ein Fortschreiten von einer Gesamteinschätzung des Korpus zur spezifischen Einzelfallanalyse ermöglichen sollte.

\subsubsection{Explorativ-vergleichende Auslegeordnung}

Mit der explorativ-vergleichenden Auslegeordnung wurde dem stufenartig konzipierten, auf Einzelartefakte zugeschnittenen Modell der Rhetorischen Designanalyse ein zusätzlicher Analyseschritt vorgeschaltet. Die Auslegeordnung, also das Auslegen und thematische Anordnen des gesamten Materials auf einer Fläche, ermöglichte nicht nur einen Überblick über das ungewöhnlich große Untersuchungskorpus, sondern schaffte auch Raum für neue Sichtweisen, die aus der theoretischen Betrachtung nicht ableitbar waren - oder vielleicht sogar mit der Theorie in Konflikt stehen würden: Indem ein exploratives Vorgehen gewählt 
wurde, bei dem ohne ein bestimmtes Frage-, Ordnungs- oder Analyseraster gearbeitet wurde, konnten die einbezogenen Gestaltungsexperten ${ }^{1}$ unvoreingenommen an das Material herantreten. Durch das Auslegen des Materials wurde es zudem möglich, Vergleiche zwischen den einzelnen Beispielen, aber auch zwischen den beiden Gestaltungsbereichen und den drei Herkunftsgemeinden zu ziehen. Die vergleichende Betrachtung ließ den relativen Elaborationsgrad der einzelnen Artefakte und ihre relative Angemessenheit hervortreten. Nicht zuletzt erleichterte die Auslegeordnung das Erfassen der vielfältigen Kriterien der Elaboration und Imperfektion, die auf die vorliegenden grafischen Artefakte angewendet wurden und die Bestimmung einer Typologie der wichtigsten Elaborationsformen.

Die explorativ-vergleichende Auslegeordnung diente somit der Beantwortung eines ausführlichen Fragekatalogs auf zwei Konkretisierungsebenen, ohne die interessierenden Fragen explizit stellen oder bereits eine bestimmte Analysemethode vorgeben zu müssen:

- Metaebene: Welches Ordnungsraster lässt sich sinnvoll auf die Fülle des vorliegenden Materials anwenden? Welche Gruppen ergeben sich? Wie sprechen ausgewiesene Gestaltungsexpertinnen und -experten über den Untersuchungsgegenstand und wie können die theoretischen Begriffe der Visuellen Rhetorik mit dem gängigen Vokabular der Visuellen Gestaltung in Übereinstimmung gebracht werden?

- Objektebene: Wie werden die einzelnen Artefakte beschrieben, zu welcher Gruppe werden sie zugeordnet und wie verhalten sie sich zueinander? Durch welche gestalterischen und elaborativen Eigenschaften zeichnet sich visuelle Kommunikation in der Lokalpolitik aus, durch welche jene in der Gemeinwesenarbeit? Welches sind typische oder häufige Elaborationsformen der visuellen Kommunikation in den untersuchten Bereichen? Welche Unterschiede und Gemeinsamkeiten lassen sich zwischen den beiden Gestaltungsbereichen sowie zwischen Stadt, Agglomeration und Land feststellen? In welches Verhältnis werden professionelles Grafikdesign und Laiengrafik gestellt? Wie wird die Angemessenheit der Artefakte bewertet? Welche Rolle spielen in den Augen der Experten Aspekte der Elaboration, insbesondere Imperfektionen, Unsauberkeiten und Fehler? Bringen die Experten bestimmte Elaborationsweisen mit Ethos-Wirkungen wie Glaubwürdigkeit, Authentizität, Seriosität oder Engagement in Verbindung?

1 Für den aufwändigen Prozess der Auslegeordnung konnten Anna Albisetti, Dozentin für Bild an der Zürcher Hochschule der Künste (ZHdK) und freischaffende Grafikerin («Peter Muster`, Zürich), und Roland Fischbacher, Studiengangsleiter BA Visuelle Kommunikation, HKB, visueller Gestalter und Kurator, gewonnen werden. 
Die beiden rund vier Stunden dauernden Expertenanalysen wurden separat und unabhängig voneinander durchgeführt. Die Aufgabe der Expertin bzw. des Experten bestand darin, das gesamte Material auf einem großen Tisch in eine ihnen sinnvoll erscheinende Auslegeordnung zu bringen und dabei «laut zu denken», also alle Zuordnungsentscheidungen und Überlegungen $\mathrm{zu}$ den einzelnen Artefakten zu kommentieren. Vorgängig wurden die rund 400 Artefakte einzig nach Herkunftsgemeinde (Stadt Bern, Agglomerationsgemeinde Zollikofen, ländliche Gemeinde Schüpfen) und Gestaltungsbereich (Lokalpolitik und Gemeinwesenarbeit) geordnet. Somit standen zu Beginn der Analyse drei mal zwei Boxen mit allen Originaldokumenten bereit. Jedes Artefakt konnte frei herausgenommen und bewegt werden. Den Experten war freigestellt, mit welchem Material sie beginnen und ob sie die beiden Gestaltungsbereiche bzw. die drei Herkunftsgemeinden getrennt oder gemeinsam beurteilen wollten. Als einzige Vorinformation wurde den Fachleuten mitgeteilt, dass es in der Studie um das Thema «Amateurgestaltung> geht.

Zur Dokumentation des Analyseprozesses wurden Tonaufnahmen gemacht und Fotos der ausgelegten Ordnungsstrukturen und ihrer Zwischenschritte erstellt. Jedes Artefakt war mit einer Nummer beschriftet, auf die sich die Experten bei der Diskussion des Beispiels beziehen sollten, um die nachträgliche Zuordnung bei der Auswertung sicherzustellen. Für die Experten war diese Untersuchungsanlage keine Standardsituation: Sowohl die große Materialfülle als auch der niedrige Elaborationsgrad der grafischen Artefakte waren für sie ungewohnt. Das Material verweigerte sich den gängigen Deutungsmustern. Bei Juryentscheidungen oder Feedbacks zu Studierendenarbeiten liegen normalerweise Einzelwerke mit hohem Professionalitäts- und Elaborationsgrad vor, wobei es um die Beurteilung der gestalterischen Konzeption und Qualität geht. Bei der Auslegeordnung hingegen waren die Evaluationskriterien offen. Um über die vorliegenden Beispiele von Laiengrafik oder durchschnittlichen Profiarbeiten zu sprechen, mussten zuerst Maßstäbe, Beschreibungsweisen und Differenzierungskriterien gefunden werden.

Zum Schluss der Auslegeordnung waren die beiden Fachpersonen angewiesen, eine Auswahl von Artefakten aus dem Gesamtmaterial zu treffen, die aus ihrer Sicht repräsentativ für das Korpus sind. Diese Expertenauswahl bildete die Ausgangslage zur Festlegung von zwölf Artefakten aus dem Korpus, welche in die Detailanalyse eingehen sollten: Kommunikationsmittel unterschiedlicher Elaborationsgrade und -typen, die jedoch exemplarisch für das Gesamtkorpus stehen können. 


\subsubsection{Designrhetorische Detailanalysen}

Zur Durchführung der Rhetorischen Detailanalysen der je sechs ausgewählten Beispiele aus Gemeinwesenarbeit und Lokalpolitik wurden vier Gestaltungsexpertinnen und -experten beigezogen. ${ }^{2}$ Die Rhetorischen Detailanalysen der zwölf Artefakte nahmen pro Experte etwa vier bis acht Stunden in Anspruch, die Resultate wurden von diesen schriftlich festgehalten. Das Analyseraster umfasste die Schritte der Rhetorischen Designanalyse in klassischer Reihenfolge: Formale Analyse, Wirkungsanalyse, Wirkregeln, Wirkungsintention und Angemessenheit, wobei zusätzlich nach Besonderheiten und gestalterischen Auffälligkeiten gefragt wurde. ${ }^{3}$ Die schriftliche Analyse wurde durch ein infografisches Analyseverfahren ergänzt, das die Faktoren Wirkung, Elaboration und Angemessenheit zusammenführen und zugleich visuell veranschaulichen sollte. Diese sogenannten ‘Tachometerskalen〉, die in einem früheren Forschungsprojekt zur Visuellen Rhetorik entwickelt wurden (vgl. Schneller 2010b, 2015; Schneller/Scheuermann 2012), erfassen Elaborationsmerkmale graduell und verbinden sie mit ihrer Angemessenheit: Auf drei Skalen pro Designvariante sollten die Experten jeweils einen Klebepunkt bei jenem Elaborationsgrad applizieren, der ihrer Meinung nach auf das jeweilige Artefakt zutrifft. Je nachdem, ob sie den Elaborationsgrad im jeweiligen Kontext für angemessen hielten, sollten sie entweder einen grünen (angemessen), einen gelben (in Frage kommend) oder einen roten Punkt (nicht angemessen) verwenden. Mit diesem Verfahren lässt sich erstens rasch überblicken, wie das Artefakt von den einzelnen Experten eingeschätzt wird. Zweitens können Gemeinsamkeiten und Unterschiede in den Expertenmeinungen sichtbar gemacht werden, indem die Klebepunkte sämtlicher Experten auf derselben Grafik angezeigt werden. Werden sämtliche Einschätzungen wiederum auf einer Skala zusammengefasst, treten drittens die für den untersuchten Gestaltungskontext geltenden Angemessenheitsbereiche zutage - unabhängig von Einzelbeispiel und -einschätzung. In der hier vorgenommenen Untersuchung sollte sich dadurch zeigen, welche Elaborationsweisen und -grade in der visuellen Kommunikation der Lokalpolitik bzw. Gemeinwesenarbeit «im grünen oder roten

2 Agnès Laube, damals Studiengangsleiterin MA Communication Design, freischaffende Grafikdesignerin, Archigrafie, Zürich. Robert Lzicar, damals Dozent für Grafikdesigngeschichte HKB, freischaffender Grafikdesigner, STVG, Zürich. Christoph Stähli Weisbrod, Dozent für Typografie HKB, freischaffender Visueller Gestalter, SetNet Bern. Esther van der Bie, Dozentin für Fotografie HKB, freischaffende Fotografin und Illustratorin.

3 Das komplette Analyseraster, wie es den Expertinnen und Experten vorgelegt wurde, ist im Anhang abgebildet. 
Bereich liegen, d.h. in diesen Gebieten generell als angemessen oder unangemessen gelten können.

Um ein übereinstimmendes Verständnis und eine einheitliche Ausführung der Analyse sicherzustellen, wurden auf dem Instruktionspapier die einzelnen Analysepunkte erklärt, mit Beispielen illustriert und die rhetorischen Fachbegriffe erläutert. Die Experten waren gebeten, alle Schritte so präzise wie möglich durchzuführen und auffällige Merkmale hervorzuheben. Um die Orientierung bei der Auswertung zu gewährleisten, wurden alle Antworten gemäß dem Nummernsystem des Analyserasters gekennzeichnet. In der Auswertung der rhetorischen Einzelanalysen wurden die vier Analyseergebnisse pro Artefakt schließlich miteinander abgeglichen und sowohl markante Übereinstimmungen als auch Abweichungen vermerkt.

\subsection{Designexperiment: Research through Design}

Um die aus der Analyse abgeleiteten Elaborationstypen und Imperfektionen in die Gestaltungspraxis zu überführen und die damit verbundenen Wirkungs- und Angemessenheitshypothesen $\mathrm{zu}$ testen, wurde eine gestalterische Versuchsanordnung entworfen, anhand deren möglichst realistische Gestaltungsaufträge formuliert und praktisch umgesetzt werden konnten. Hierbei sollte die grafische Gestaltung selbst - sowohl der Prozess als auch das Produkt - als Forschungsund Erkenntnismittel genutzt werden. Dieses «Designexperiment〉 stützt sich auf die Methode des «Research through Design〉, die von Christopher Frayling (Frayling 1993) am «Royal College of Art London erstmals benannt und von Designforschenden wie Kees Overbeecke (Overbeeke 2006) und Alain Findeli (Findeli et al. 2008) weiter ausformuliert wurde (vgl. auch Mareis 2011: 62-66). Bis heute ist diese Methode nicht einheitlich definiert. «Erforschen durch Gestalten〉 bedeutet im Fall der hier gewählten experimentellen Anordnung, Hypothesen zu testen, die aus der Rhetorischen Designanalyse abgeleitet wurden, indem Designentwürfe nach bestimmten Vorgaben ausgearbeitet werden. Durch die gezielte Erarbeitung unterschiedlicher Varianten desselben Designprodukts mit je spezifischen Elaborationsmerkmalen und Imperfektionen sollte eine optimale Vergleichbarkeit und Überprüfbarkeit der Objekte und ihrer Wirkungen ermöglicht werden. 


\subsubsection{Experimentelle Gestaltung von Designvarianten unterschiedlicher Elaborationsgrade}

Als Ausgangspunkte für das Gestaltungsexperiment dienten drei grafische Kommunikationsmittel, die zuvor in der Rhetorischen Detailanalyse untersucht worden waren. Gemäß einem «Briefing〉 wurden je zwei Designvarianten dieser drei Originalartefakte mit unterschiedlichem Elaborationsgrad ausgestaltet wobei das Briefing einem realen Gestaltungsauftrag möglichst nahe kommen sollte. ${ }^{4}$ Für die am wenigsten elaborierte Variante des Originals aus dem Bereich Lokalpolitik musste der Elaborationsgrad auf ein semi-professionelles Niveau herabgesetzt werden, was für die Profigestalterinnen ungewohnt, aber machbar war - weshalb auch darauf verzichtet wurde, hierfür extra einen gestalterischen Laien anzufragen. Auch die Tatsache, dass nicht mit den realen Auftraggebern kommuniziert werden konnte, trug zu einer gewissen Künstlichkeit der Situation bei. Dennoch erlebten die Gestalterinnen den Prozess und die dabei entstandenen Produkte insgesamt als ausreichend an die gestalterische Arbeitsrealität angepasst. Zusammen mit den Originalen entstanden so insgesamt je drei Artefakte, welche dasselbe Thema behandelten und dieselben Inhalte repräsentierten, die jedoch in unterschiedlichen Graden elaboriert bzw. unterschiedlich professionell umgesetzt waren.

\subsubsection{Wirkungsstudie: Leitfadenbasierte persönliche Befragung}

Zur Überprüfung der in der Auslegeordnung und Rhetorischen Designanalyse gewonnenen Thesen über die Elaborationstypen und Wirkungen wenig elaborierter Gestaltung im Kontext der Gemeinwesenarbeit und lokalpolitischen Kommunikation wurden die gestalteten Designvarianten aus dem «Research through Design> zwanzig Versuchspersonen vorgelegt. Anders als bei der zuvor durchgeführten Rhetorischen Designanalyse sollten hier Personen befragt werden, die keine besondere gestalterische Expertise mitbringen und als Zielpublikum der gestalteten Kommunikationsmittel in Frage kämen. Die Wirkungsstudie beruht auf einer leitfadenbasierten, rund 50 Minuten dauernden Eins-zu-eins-Befragung von Probanden verschiedener Ausbildungs- und Berufsprofile sowie unterschiedlicher Altersgruppen aus städtischen, stadtnahen und ländlichen Regionen des Kantons Bern. Da die gestalteten Designvarianten auch Unterschiede

4 Die Gestaltungsaufgaben wurden durch die Grafikerinnen Anna Albisetti, welche bereits bei der Auslegeordnung involviert gewesen war, und Nathalie Matter vom Atelier «Peter Muster», Zürich, übernommen. 
in Haptik, Format, Dramaturgie, Material- und Druckqualität aufwiesen, um die Unterschiede im Elaborationsgrad auf allen Ebenen der grafischen Gestaltung zur Geltung zu bringen, kam nur eine persönliche Befragung in Frage, bei der die Probanden mit den Artefakten als physischen Objekten in Kontakt kommen konnten. Eine Onlinebefragung hätte zwar mit wenig Aufwand eine weit größere Probandenzahl zugelassen und so eine höhere Repräsentativität erreicht, doch wären aufgrund der digitalen Wiedergabe zentrale Elaborations- und Wirkungsunterschiede nicht messbar gewesen. Auf eine Videoaufzeichnung wurde verzichtet, da ein Raster zur schriftlichen Erfassung entwickelt werden konnte, das eine eindeutige Zuordnung der Antworten zu den einzelnen Artefakten zuließ. Für die Befragungen wurde ein randomisierter Ablauf gewählt, d.h. jede Person beurteilte die jeweils drei Artefakte einer Gruppe in wechselnder Reihenfolge, um eine Beeinflussung der Wahrnehmung durch gleichbleibende Variantenabfolgen bzw. vorangehende Artefakte auszuschließen. Auf die Einzelanalyse folgte eine Vergleichsphase, bei der die drei Varianten einander gegenübergestellt wurden. Ein Leitfaden ${ }^{5}$ sollte gewährleisten, dass alle Probanden zu jeder Variante dieselben Fragen beantworteten, jedoch offen antworten und spontane Beobachtungen vorbringen konnten. $\mathrm{Zu}$ allen neun Designvarianten, d.h. auch zu den Originalen aus dem Untersuchungskorpus, wurden sechs Fragen gestellt: Was ist Ihr erster Eindruck, wenn Sie diesen Flyer oder Prospekt sehen? Wie wirkt er konkret auf Sie? Auf welche Gestaltungsmerkmale führen Sie die Wirkung zurück? Wird für Sie rasch klar, wofür dieser Flyer/Prospekt wirbt oder um welches Angebot es geht? Ist dieser Flyer/Prospekt Ihrer Meinung nach professionell gestaltet? Halten Sie diesen Flyer/Prospekt für angemessen gestaltet?

Sobald die drei Varianten eines Bereichs beurteilt worden waren, wurden diese miteinander verglichen. Dabei sollten die Probanden angeben, welche der Varianten sie im Verhältnis zu den anderen Varianten für passender oder weniger passend, für professioneller oder weniger professionell hielten. Zum Abschluss der Befragung wurden die soziodemografischen Daten der Testpersonen erhoben (Geschlecht, Alter, Ausbildung, Beruf, Wohnort, Nationalität) und ihre Nähe zu den beworbenen Absendern und Angeboten eruiert. Dadurch sollten mögliche Sympathie -oder Ablehnungseffekte eingeordnet werden können, welche die Beurteilung der Artefakte beeinflussen könnten.

5 Das komplette Befragungsraster, das für die Wirkungsstudie verwendet wurde, ist im Anhang abgebildet. 